\title{
COMENTARIO AL TRABAJO DE JOYCE SLOCHOWER "NO SE LO DIGAS A NADIE"1
}

\author{
Sandra Toribio Caballero² \\ IPR, IARPP (Madrid)
}

\begin{abstract}
Las violaciones de límites invaden todas las comunidades profesionales, pero resultan especialmente dramáticas en la nuestra: en psicoterapia, el trabajo está basado en la confianza básica y nuestro código deontológico incluye el no llevar a cabo prácticas que atenten contra la libertad e integridad física y psíquica de las personas. Pero, ¿qué hacer cuando conocemos que un determinado profesional ha abusado de un paciente? ¿Se pueden evitar o prevenir estas estas violaciones de límites? En el presente artículo se reflexiona sobre el texto de Slochower, incluyendo una mirada desde la perspectiva de género.
\end{abstract}

Palabras clave: Violaciones de límites sexuales, abusos en consulta, perspectiva de género, \#MeToo.

Boundary violations invade every professional community, but these are especially dramatic in ours: in Psychotherapy, work is based on basic trust, and our deontological code includes not to carry out practices that attempt to the freedom, physical and psychological integrity of the people we work with. But, what can be done once we find out that a certain psychotherapist has abused a patient? Can we avoid or prevent these boundary violations? In the present article we reflect on Slochower's paper, including gender perspective.

Key Words: Sexual boundary violations, abuse in the consulting room, gender perspective, \#MeToo.

English Title: Commentary to Slochower's paper "Don't tell anyone"

Cita bibliográfica / Reference citation:

Toribio Caballero, S. (2018). Comentario al trabajo de Joyce Slochower "No se lo digas a nadie". Clínica e Investigación Relacional, 12 (3): 436-443. [ISSN 1988-2939] [Recuperado de www.ceir.info ] DOI: $10.21110 / 19882939.2018 .120302$

\footnotetext{
${ }^{1}$ Comentario leído tras la conferencia de la prof. Slochower el 29/07/18 en el Instituto de Psicoterapia Relacional, Madrid.

${ }^{2}$ Psicoterapeuta infanto-juvenil y de adultos. Psicóloga General Sanitaria. Licenciada en Psicología por la Universidad Complutense de Madrid. Especialista en Psicoterapia Psicoanalítica Relacional y en la aplicación del Test Rorschach. Máster en Mujeres y Salud (UCM); actualmente trabajando en su Tesis Doctoral sobre Psicopatología y Género. Psicoterapeuta reconocida por la FEAP (Federación Española de Asociaciones de Psicoterapeutas). Miembro del Instituto de Psicoterapia Relacional, de la IFP (International Federation for Psychotherapy) y de la IAPSP (International Association for Psychoanalytic Self Psychology). Presidenta del Comité de Candidatos de IARPP (International Association for Relational Psychoanalysis and Psychotherapy).
} 


\section{INTRODUCCIÓN}

Algo está cambiando en el mundo. Las noticias (y sentencias) sobre violaciones en grupo indignan, repugnan y, sobre todo, ahora más que nunca, movilizan. El pasado 8 de marzo, Día Internacional de la Mujer, vivimos en Madrid una manifestación como nunca antes en la historia de ese día. El movimiento \#MeToo, que englobaba a mujeres que pusieron palabras a los abusos cometidos por Harvey Weinstein, poderoso productor de Hollywood, no dejó indiferente a nadie. Pero ¿qué pasa con los abusos cometidos dentro de nuestro submundo, el psicoanalítico?

Sin duda, las violaciones de los límites invaden todas las comunidades profesionales (quizás podríamos decir todas las comunidades, a secas), pero no podemos obviar que resultan especialmente dramáticas en la nuestra, donde nuestro trabajo está basado en la confianza básica y en nuestro código deontológico está incluido el no llevar a cabo prácticas que atenten a la libertad e integridad física y psíquica de las personas. Entre los objetivos de nuestro trabajo deben estar siempre el bienestar, la salud y la calidad de vida. Somos una profesión de cuidados.

El texto de la profesora Slochower es importante, impactante, y especialmente necesario, ya que nombra algo que pertenece a nuestro saber compartido como psicoanalistas... y que es a la vez un saber callado, soterrado.

\section{ABUSOS EN LA CONSULTA}

Cuando leí el artículo de Muriel Dimen (2011) "Lapsus Linguae, ¿o un desliz de la lengua? Un caso de abuso sexual dentro de un tratamiento analítico y sus consecuencias personales y teóricas" por primera vez, quedé tremendamente impactada. ¿Acaso esas cosas pasaban en el mundo psicoanalítico? Sí, de acuerdo, todos conocemos algún psicoterapeuta "un poco" psicópata ... pero ¿besar con lengua a una paciente... y hacer como que no ha pasado? ¿Cómo seguir después en análisis con esa persona? Hablé del artículo con varias colegas, y enseguida descubrí que eso no era algo que pasara sólo en Nueva York hace alguna década. También pasaba mucho más cerca, y de forma mucho más reciente. Desde luego no es lo habitual, pero sí era más frecuente de lo que yo nunca hubiera imaginado. Sentí que mi profesión, a la que tanto quería, tenía de repente zonas turbias y oscuras...

Slochower (2017) habla del difícil papel que tienen aquellas personas que son testigos indirectos de las violaciones. ¿Qué se puede hacer si quien lo sufre en primera persona no quiere "denunciar" (de la forma en la que sea)?. "No sabemos qué decir, a quién contárselo, 
o qué hacer. Tremendamente alterados, nos sentimos indefensos y con frecuencia inmovilizados", dice Slochower (2017). Pero, acaso, ¿no tenemos el deber moral de intervenir?

Se pregunta también qué pasaría si, al denunciar, visibilizar o dar voz, fuera peor el remedio que la enfermedad. ¿Qué pasará con aquellas/os pacientes, supervisandos/as o alumnos/as a los que esa persona sí que haya podido ayudar?

Como señala Slochower, la realidad es que cuando estas informaciones nos llegan, aunque sea en forma de rumores, dejan de serlo y pasan a ser datos/hechos cuando son conocidos por varias personas. Es entonces cuando la comunidad psicoanalítica (o de un determinado ámbito o instituto) se convierte en testigo. Me lleva a pensar a la teoría de la psicología social sobre la difusión de la responsabilidad (Worchel, 2003): cuando hay presente una sola persona como testigo, toda la ayuda debe venir de él o de ella - no queda más que intervenir. Pero cuando hay varios/as observadores/as, la ayuda no se centra en ninguno, si no que se divide entre todos/as, y como consecuencia o resultado, no ayuda nadie.

Slochower también apunta a que el reconocimiento de estos hechos hace tambalear o termina por derribar nuestras idealizaciones, tan necesarias cuando estamos en formación, en supervisión o en análisis. Se resquebrajará así la idea que teníamos de un determinado profesor, supervisor o terapeuta. Dar los rumores/hechos por buenos, nos pone también en riesgo de perder algo que sentíamos que teníamos.

Las violaciones de los límites sexuales, nos dice Slochower (2017), son como fantasmas, a los que aún no hemos analizado. Tal y como nos dice la teoría del trauma, lo verdaderamente traumático no es el suceso traumático en sí mismo, sino el hecho de que éste sea cometido por aquel/aquella que se supone nos debe cuidar. Es quien nos debe proteger quien nos hace el daño. Desde ahí se puede entender mejor lo difícil (o lo imposible) que puede resultar el romper el vínculo una vez traspasados los límites o cometido el abuso.

Hay algo de la trasgresión de límites que también, como señala Slochower, nos toca, y es que nos conecta con nuestras propias fantasías, con el morbo. ¿Qué pasaría si nosotros/as, como terapeutas, nos viésemos en esa situación, sintiendo una atracción que se nos antojara incontrolable por un/a paciente? Seguramente sería una minoría de las personas que lean éste texto quienes responderían "a mí me ha pasado" o "podría pasarme a mí" - y en realidad, quizás sea mejor así: que sea una minoría lo más cercana al "cero" posible, ya que esto nos daría sensación de seguridad y protección. Estaríamos a salvo, como si fuera un escudo protector o un mecanismo de defensa contra ese posible traspasar los límites. 
Pensar que estamos a salvo de traspasar este $u$ otro tipo de límites que a priori (y afortunadamente) se nos antojan aborrecibles, es quizás no contemplar la posibilidad de que, quizás, en el algún momento de nuestras carreras profesionales, puedan juntarse circunstancias que nos hagan actuar como nunca pensamos que lo haríamos. Eso no será lo peor. Lo peor será que pase repetidamente, que no lo podamos pensar, que no lo podamos hablar o compartir, ... Me vienen algunas frases del saber popular a la cabeza: "No hay nada más castigado que la boca", "Nunca digas de esta agua no beberé", o "Quien esté libre de pecado que tire la primera piedra".

Como decíamos al principio, podría parecer que las violaciones sexuales y de límites no tienen lugar... pero en realidad no es que no existan, es que no se habla de ellas, como pasa en muchas ocasiones con los abusos. $Y$ es que, tal y como señala Dimen (2011), para nosotros, como psicoterapeutas, las violaciones de límites por parte de otros colegas son tabú, como lo es un "buen" secreto de familia. Slochower (2017) cuenta como, en un seminario que imparte, en un grupo de diez personas, hablaron - sin llegar a nombrarlo de un analista de su instituto que había traspasado los límites; sí que pudieron, sin embargo, hablar de "otro analista que había traspasado los límites... porque pertenecía a otro instituto de formación.

Los secretos, además, pueden dar cierto estatus y sensación de pertenencia a un club "exclusivo". La información es poder, y ese "yo sé algo que tú no sabes" puede estar en relación con el "morbo" del que hablábamos antes.

¿Podemos incluir las violaciones de los límites sexuales en la categoría de fallas analíticas, de fracasos del terapeuta? A lo mejor sí... pero seguramente también son algo que va más allá, indudablemente más grave que otros tipos de rupturas en el encuadre. Las personas que consultan buscan un espacio y lugar seguro donde otro va a velar por su seguridad, y el abuso de poder basado en la asimetría de la relación hace saltar por los aires esa confianza básica de la que hablábamos, que debe estar siempre en la base de la relación terapéutica.

Esto recuerda al movimiento feminista \#MeToo, que nombrábamos al principio, y que destapó los numerosísimos abusos del productor de cine Harvey Weinstein. Muchas de las mujeres que habían sido acosadas por él tenían miedo a hacerlo público porque, ¿quién iba a creerlas a ellas? ¿Acaso no las acusarían de buscar la fama, de buscar un papel en alguna película? Él, un hombre tremendamente poderoso en la industria del cine; y ellas, chicas jóvenes en busca de una oportunidad.

Por cierto, de forma similar a la duda que nos planteaba el denunciar (de la forma en la que fuera) a una persona de nuestra comunidad de la que tengamos constancia que ha cometido abusos porque eso pondría en peligro a otras personas a las que sí ha podido 
ayudar, el caso Harvey Weinstein también trajo a la palestra el debate sobre si deberíamos seguir viendo películas o series de actores que (supuestamente) hayan abusado de actores, actrices o allegados (véase, Woody Allen o Kevin Spacey).

Hace poco leí un artículo sobre qué había sido de Mónica Lewinsky, aquella becaria que guardó un vestido con el semen del entonces presidente de los Estados Unidos, Bill Clinton. Pero, ... un momento, ¿acaso ese episodio no debería haber quedado "archivado" en mi memoria como el caso donde un presidente de Estados Unidos tuvo una relación extramatrimonial (ella era soltera, aunque en realidad también desconocemos los entresijos de si la relación matrimonial entre Bill y Hilary era de naturaleza abierta - por ejemplo) con una chica joven, contratada como becaria? ¿Y si le diéramos la vuelta al titular, donde habláramos de una víctima - o superviviente - de una situación de abuso? De nuevo: Hombre mayor poderoso - chica joven en prácticas. Mónica Lewinsky reportó haber sufrido Trastorno de Estrés Post-Traumático tras el juicio ${ }^{1}$.

(Como nota: sin duda los abusos son siempre algo traumático, pero quisiera recalcar que nuestra profesión tiene algo específico que otras - como el mundo del cine - no tienen, y es que como decíamos al principio, la nuestra es una profesión de cuidados, nosotros tenemos un código deontológico, el mundo del cine no lo tiene).

Slochower habla en su texto también de las justificaciones que podemos terminar encontrando para esos abusos cometidos por terapeutas. En muchas ocasiones resulta difícil, como decíamos, creer a la víctima, que suele ser mujer. Aparecen entonces las racionalizaciones y dudas sobre lo que sabemos: "No será para tanto", "Es adulta, puede defenderse por sí misma" ...

\section{LO IMPRESCINDIBLE DE LA PERSPECTIVA DE GÉNERO}

Los abusos de terapeutas a pacientes, además de ser algo traumático, llevan intrínseco otro denominador común: suelen ser hombres-terapeutas los que abusan de mujerespacientes. Por eso, en este área (como en todas las relacionadas con la salud), resulta imprescindible que los/las terapeutas tengan/tengamos perspectiva de género y podamos trabajar incluyéndola. El poder, en nuestra sociedad, es primordialmente masculino (Slochower explica que en el artículo habla de "Él" para referirse al perpetrador de la violación porque normalmente son hombres). Sí, inevitablemente la relación terapéutica es asimétrica (aunque para nosotros como relacionales no lo sea tanto), y el/la terapeuta tiene - por el hecho de serlo - una cierta posición de poder. Pero esa distancia será 
ineludiblemente más grande si el terapeuta es un hombre, ya que tendrá "doble" poder: por ser terapeuta, y por ser hombre.

Además, los abusos en la consulta replicarían los patrones-roles-posiciones que en nuestra cultura/sociedad se corresponden típicamente con lo masculino (lo activo, lo relacionado con el placer y el disfrute; expresa y ejerce su deseo) y con lo femenino (lo pasivo, lo relacionado con el sufrimiento; la mujer como objeto de deseo). Si el terapeuta/hombre invade con su deseo el espacio terapéutico, ¿dónde queda el espacio para que se despliegue el deseo de la paciente/mujer?

Dimen (1991) nos recuerda que "todos los géneros son creados como iguales, pero algunos son más iguales que otros".

Resulta inevitable pensar que con las situaciones de abuso en la consulta se reproduce la dinámica Amo-Esclavo de Hegel, que tanto ha trabajado Benjamin, y cómo enseguida vienen a la cabeza series televisivas actuales como "El cuento de la criada".

\section{QUÉ SE PUEDE HACER: REFLEXIONES Y PERSPECTIVAS FUTURAS}

Algo maravilloso pasa al leer el artículo: parece que está vivo, que se mueve. La autora nos lleva a su propio proceso reflexivo-introspectivo de escritura y creación... y dan casi ganas de hacer algo.

Pero ¿qué podemos hacer ante este panorama? ¿se pueden prevenir los abusos? ¿o son algo con lo que hay que contar, un punto negro representado por una minoría?

Slochower (2017) se pregunta qué hemos hecho para terminar con este problema, que es parte de nuestra profesión desde el principio de los tiempos. Quizás el enorme contrasentido es que nosotros, que trabajamos poniendo en palabras lo que no puede decirse ( $y$ animando a nuestros/as pacientes a que lo hagan), que sabemos bien de lo traumático de los silencios y de lo innombrable, sentimos un miedo atroz, que nos paraliza, cuando nos toca a nosotros/as hablar de abusos. Hay que tener en cuenta también que no contamos con un sistema legal que, a priori, proteja a las víctimas: podemos no tener claro cómo o dónde hacer la denuncia.

Señala Slochower, además, que quizás "en nuestra comunidad hemos fallado en restringir las violaciones porque estamos implicados en ellas". Propone que tenemos que hacer algo como comunidad y habla de la justicia restaurativa como posibilidad.

Pero ¿se pueden rehabilitar terapeutas (mayoritariamente hombres) que hayan transgredido límites (con, mayoritariamente, mujeres) ... sin pensar que parte de su 
conducta o comportamiento tiene que ver con el hecho de ser hombres y su connotación en nuestra sociedad/cultura? ¿Se pueden "rehabilitar" personas que hayan abusado/violado a mujeres? Esta pregunta nos podría llevar - aún desviándonos un poco del tema que nos ocupa - a otra: ¿se pueden rehabilitar maltratadores dentro de una cultura machista? La realidad es que vivimos en la cultura de la violación, así nombrada desde hace no tanto por los medios de comunicación. Y la realidad es que, a día de hoy, no existen experiencias exitosas de rehabilitación de maltratadores, tal y como señala la periodista y escritora experta en género Nuria Varela.

Obviamente, dentro del campo de la prevención, como psicoanalistas pensaremos enseguida en la importancia del análisis, de la supervisión, y de la formación, ... sí, eso ya nos lo sabemos. Pero quizás también deberíamos incluir la importancia de tener cerca amigos/as, compañeros/as en la profesión, que tantas veces nos ayudan (y a los/las que ayudamos) a sostenernos (a sostener). Quizás tenemos que buscar (si es que no contamos ya con ellos) con espacios seguros entre iguales: indudablemente da menos pudor hablar de nuestras fallas con colegas que con supervisores/as o profesores/as. Esa "supervisión informal", donde quizás nos podamos sentir más libres, puede suponer un escudo protector "extra".

Artículos como el de Slochower o Dimen son necesarios, así como los de Gabbard. Libros como "La imposibilidad del sexo. Historias de la relación íntima entre terapeuta y paciente" de Susie Orbach son tremendamente valiosos, especialmente por la honestidad con la que la autora habla de sus propios sentimientos con sus pacientes. $Y$ no me refiero a los que tenemos ya muy manidos en nuestros textos: simpatía, afecto, enfado, irritación, ... No. Me refiero a otros de los que normalmente nos cuesta más escribir, esos que son políticamente menos correctos: excitación, amor, deseo, atracción, ... ganas de acostarse con.

Quizás si poco a poco nos atrevemos a hablar (y a escribir) más de esa otra clase de sentimientos, podamos abrir la veda y el espacio a detectar y prevenir el ir un paso más allá en la trasgresión de límites.

Pero la realidad es que seguirá habiendo terapeutas que abusen de sus pacientes.

Como decíamos al principio, el momento actual - de cambios y denuncia social acompaña: estamos ante lo que ya muchos/as llaman la Cuarta Ola Feminista, donde se da voz y visibiliza mucho más que antes al malestar de las mujeres. Quizás ahora el contexto ayude y podamos unirnos a las voces de denuncia respecto a los abusos cometidos dentro del mundo psicoanalítico. Aunque el marco legal y la interpretación que puedan hacer jueces y juezas del mismo no esté siempre a la altura de lo que demanda gran parte de la 
sociedad (caso La Manada), tendremos que buscar ámbitos donde solucionar, como comunidad, lo que tiene que ver con nuestras propias transgresiones.

Quizás en este darle voz todos y todas podemos poner nuestro granito de arena. Si empezamos por reconocer, por visibilizar y por hablar de nuestras propias fallas, a comentar y compartir nuestras fantasías (en ambientes que sintamos seguros), podremos poco a poco integrarlo como algo que pasa y es inevitable.

Necesitamos el reconocimiento del que habla Benjamin, no sólo a nivel individual, sino a nivel grupal o comunitario, para intentar ayudar así la reparación del daño causado. Hacerlo público y compartirlo. $Y$ dar quizás también oportunidad a que los perpetradores se rehabiliten (aquellos que puedan).

Desde luego, seguirá habiendo personas (hombres) que abusen de pacientes, pero poco a poco, las mujeres que han sido víctimas (y son supervivientes) y la comunidad analítica podrán alzar la voz sin miedo (la realidad es que cada vez hay menos miedo) y decirles, recordarles, que no están solas.

\section{REFERENCIAS}

Código Deontológico del Psicólogo. Consultado el 26/06/18 en: http://www.copmadrid.org/webcopm/recursos/codigodeontologicojunio2010.pdf

Dimen, M. (1991). Deconstructing difference: Gender, splitting and transitional space. Psychoanalytic Dialogues, 1, 335-352.

Dimen, M. (2011). Lapsus linguae, or a slip of the tongue? A sexual violation in an analytic treatment and its personal and theoretical aftermath. Contemporary Psychoanalysis, 47 (1), 3579.

Gabbard, G. O. \& Lester, E. P. (1995). Boundaries and boundary violations in Psychoanalysis. Washington: American Psychiatric Publishing.

Orbach, S. (2000). The impossibility of sex. Stories of the intimate relationship between therapist and patient. Nueva York: Touchstone.

Slochower, J. (2017). Don't tell anyone. Psychoanalytic Psychology, 34(2), 195-200.

Worchel, P., Cooper, J., Goethals, G. R., y Olson, J. M. (2003). Psicología Social. Madrid: Thomson.

Original recibido con fecha: $\quad 30 / 6 / 2018 \quad$ Revisado: 27/9/2018 Aceptado: 30/102018 NOTAS:

\footnotetext{
${ }^{1}$ https://www.telegraph.co.uk/news/2018/02/26/monica-lewinsky-says-victim-abuse-authority-course-affairpresident/
} expresa. Este material es para uso científico y profesional exclusivamente y puede contener información clínica sensible. Los editores no se responsabilizan de los contenidos de los autores. Dirigir las consultas sobre derechos y autorizaciones a ceir@psicoterapiarelacional.es 